\title{
PERFIL EPIDEMIOLÓGICO DO HOMEM COM CÂNCER DE PRÓSTATA ATENDIDO EM UM HOSPITAL UNIVERSITÁRIO*
}

Marina Viana Fernandes', Júlia Trevisan Martins², Alexandrina Aparecida Maciel Cardelli³, Sonia Silva Marcon ${ }^{4}$, Renata Perfeito Ribeiro: Enfermeira ${ }^{4}$

${ }^{1}$ Enfermeira. Universidade Estadual de Londrina. Londrina-PR-Brasil.

Enfermeira. Doutora em Enfermagem Fundamental. Universidade Estadual de Londrina.

${ }^{3}$ Londrina-PR-Brasil.Enfermeira. Doutora em Saúde Pública. Universidade Estadual de Londrina. Londrina-PR-Brasil.

${ }^{4}$ Enfermeira. Doutora em Enfermagem. Universidade Estadual de Londrina. Londrina-PR-Brasil.

RESUMO: Pesquisa epidemiológica, descritiva com recorte transversal teve por objetivo traçar o perfil epidemiológico de homens com câncer de próstata assistidos em Hospital Universitário do Sul do Brasil. Os dados foram coletados por meio de entrevista a 54 homens, empregando-se formulário estruturado abordando características sociodemográficas, comportamento de saúde e história da doença. Para a análise dos dados utilizou-se o Programa SPSS versão 19. Os resultados apontaram idade média de 74,8 anos, predomínio de brancos; baixa escolaridade; aposentados e coabitação familiar; foram identificados como comportamentos de risco para o câncer de próstata: alimentação inadequada; tabagismo; ingestão de bebida alcoólica; e tempo médio da procura por cuidados superior a 30 dias. Estratégias devem ser adotadas por gestores públicos e equipe multiprofissional para informar o homem sobre a importância de atitude positiva de prevenção.

DESCRITORES: Saúde do homem; Neoplasias da próstata; Enfermagem.

\section{EPIDEMIOLOGICAL PROFILE OF MEN WITH PROSTATE CANCER ATTENDED IN A TEACHING HOSPITAL}

\begin{abstract}
This epidemiological, descriptive, crosssectional research aimed to outline the epidemiological profile of men with prostate cancer assisted in a Teaching Hospital in the south of Brazil. The data were collected through interviews with 54 men, using a structured questionnaire addressing socio-demographic characteristics, health behavior and history of the disease. The SPSS Program, version 19, was used for analysis of the data. The results indicated a mean age of 74.8 years old; the prevalence of Caucasians; low educational level; retirees and living with family members. Behaviors of risk for prostate cancer were identified: poor food; smoking; alcoholic drinks; and a mean time taken to seek treatment of over 30 days. Public managers and the multi-professional team must adopt strategies in order to inform men about the importance of a positive preventive attitude.
\end{abstract}

DESCRIPTORS: Men's health; Prostatic neoplasm; Nursing.

*Resultado extraído de Dissertação de Mestrado em Enfermagem - Universidade Estadual de Londrina, apresentada em 2011.

\section{PERFIL EPIDEMIOLÓGICO DEL HOMBRE CON CÁNCER DE PRÓSTATA ATENDIDO EN UN HOSPITAL UNIVERSITARIO}

RESUMEN: Investigación epidemiológica, descriptiva con corte transversal que tuvo la finalidad de trazar el perfil epidemiológico de hombres con cáncer de próstata asistidos en Hospital Universitario del Sur de Brasil. Los datos fueron recogidos por medio de entrevista con 54 hombres, utilizándose el formulario estructurado que cuestionaba las características sociodemográficas, comportamiento de salud y historia de la enfermedad. Para el análisis de los datos, fue usado el Programa SPSS versión 19. Los resultados apuntaron edad media de 74,8 años, predominio de blancos; poca escolaridad; jubilados y que vivían con familiares; fueron identificados como comportamientos de riesgo para el cáncer de próstata: alimentación inadecuada; tabaquismo; ingestión de bebida alcohólica; y tiempo medio de busca por cuidados superior a 30 días. Estrategias deben ser adoptadas por gestores públicos y equipo multiprofesional para informar al hombre sobre la importancia de actitud positiva de prevención. DESCRIPTORES: Salud del hombre; Neoplasias de la próstata; Enfermería.

\section{Autor Correspondente:}

Recebido: $18 / 04 / 2013$ Finalizado: 10/03/2014

Sonia Silva Marcon

Universidade Estadual de Londrina

Rua Santos, 488 - 86020-040 - Londrina-PR-Brasil

E-mail: perfeito@sercomtel.com.br 


\section{INTRODUÇÃO}

O Câncer de Próstata (CaP) é o mais comumente diagnosticado entre homens. Foram registrados 899.000 casos novos e 258.000 mortes em 2008, e as estimativas apontam aumento de 1,7 milhões de novos casos e de 499.000 mortes até 2030, o que demonstra que as taxas de mortalidade continuarão inalteradas no futuro ${ }^{(1)}$.

Em 2012, em todo o mundo, ocorreram 14,1 novos casos de câncer, 8,2 milhões de morte e 32,6 milhões de pessoas vivendo com esse agravo. Destes, 8 milhões de casos novos, 5,3 milhões de mortes por câncer ocorreram nas regiões menos favorecidas. A taxa global de sua incidência, por idade padronizada é quase $25 \%$ maior em homens do que em mulheres. Sendo que essas taxas variam de 79 por 100.000 na África do Sul a 365 por 100.000 na Austrália/ Nova Zelândia, que representa um índice perigoso e preocupante, nessa última região ${ }^{(2)}$.

Em relação à mortalidade há menor variabilidade regional do que na incidência, sendo taxas $15 \%$ maiores nas regiões mais desenvolvidas nos homens. Nos homens as taxas mais elevadas ocorrem na Europa Central e Oriental (173 por 100.000) e menor na África Ocidental (69 por 100.000) $)^{(2)}$.

Na América do Sul, em 2008, o CaP foi a primeira causa de morte entre os homens ${ }^{(3)}$. No Brasil, a estimativa deste câncer, para os anos de 2012 e 2013, foi de 60 mil novos casos, ficando na segunda posição dos cânceres em geral, para ambos os sexos, e na primeira para os homens ${ }^{(2)}$. Para 2014 estima-se 68.800 casos novos de CaP e a maior incidência estimada é na da região Sul (90 por 100.000 habitantes) $)^{(4)}$.

São vários os fatores predisponentes para o $\mathrm{CaP}$, entre eles estão: a idade avançada, raça negra/etnia, história familiar da doença, estilo de vida, hábitos alimentares, sedentarismo, obesidade e consumo de tabaco ${ }^{(5-8)}$. O CaP é passível de prevenção e pode ser evitado se for diagnosticado precocemente e atualmente recomenda-se que o rastreamento seja oferecido ao homem a partir dos 50 anos e com expectativa de vida maior que 10 anos e a homens de alto risco e muito alto risco a partir de 45 e 40 anos respectivamente, após a apresentação dos benefícios e riscos ao indivíduo que deve fazer a sua escolha ${ }^{(9)}$. Os altos índices da doença são atribuídos ao fato de que a busca por cuidados preventivos de saúde não é prática comum entre a população masculina. O homem normalmente, não reconhece, desvalorizando as próprias necessidades de saúde ${ }^{(10-12)}$. Pode-se, inclusive, afirmar que a identidade masculina constitui um fator de risco para a saúde ${ }^{(12-14)}$, uma vez que o homem se torna vulnerável quando aceita, sem reflexão, padrões de gênero constituídos cultural e socialmente.

Por outro lado, apesar da disponibilização do exame de prevenção do CaP pelo sistema público de saúde, a demanda é insignificante. Este grupo ainda não adquiriu o hábito de buscar o serviço de saúde, mesmo na vigência de queixas ${ }^{(11)}$. Neste sentido, a efetivação de ações de atenção à saúde do homem, voltadas à reflexão sobre a influência desses valores culturais na prevenção de agravos, como diagnóstico, tratamento, reabilitação, manutenção e proteção da saúde, representam um desafio ${ }^{(14)}$. A Política Nacional de Atenção Integral ao Homem ${ }^{(15)}$ reconhece os agravos à saúde dessa população como problema de saúde pública, por este motivo, determinou que fossem realizadas mais pesquisas na área.

Assim sendo, o presente estudo é de fundamental importância, visto que, conhecer o estilo de vida e os hábitos de procura por cuidados de saúde pelos homens pode subsidiar planejamentos estratégicos para a promoção da saúde, prevenção da doença, diminuição de agravos, tratamento e recuperação e por consequência melhorar a qualidade de vida destes homens e de suas famílias.

A pesquisa vem ao encontro da lei $n$. 10.289 , de 20 de setembro de $2001^{(16)}$ que preconiza a implementação do Programa Nacional de Controle do Câncer de Próstata, e estabelece que sejam desenvolvidas atividades como: campanha institucional nos meios de comunicação, com mensagens sobre o que é o câncer de próstata e suas formas de prevenção; parcerias com as Secretarias Estaduais e Municipais de Saúde, universidades, sociedades civis organizadas e sindicatos, organizando-se debates e palestras sobre a prevenção e formas de combate dessa doença.

Diante das considerações anteriores, o objetivo desta pesquisa foi traçar o perfil epidemiológico de homens com CaP atendidos em um Hospital 
Universitário do Sul do país.

\section{MÉTODO}

Pesquisa do tipo epidemiológica, com recorte transversal e abordagem quantitativa realizada entre abril e outubro de 2011. A população do estudo foi constituída por 54 indivíduos, de um total de 70 homens acometidos por CaP cadastrados e acompanhados no ano de 2010 e 2011 no ambulatório de Uro-oncologia do Hospital Universitário de Londrina. Os critérios de inclusão foram: diagnóstico de CaP há mais de 6 meses e apresentar condições físicas e cognitivas para a participação nas entrevistas.

Para a coleta de dados utilizou-se um questionários com perguntas fechadas e abertas e técnica de entrevista que foi gravada e transcrita para um formulário estruturado contendo dados de identificação; variáveis sociodemográficas (idade, procedência, raça, religião, escolaridade, condição de trabalho atual e pregressa, proveniência da renda); estilo de vida (condição de coabitação, quem cuida, tipo de cuidado, hábitos alimentares, consumo de bebida alcoólica, tabaco e drogas, atividade física e de lazer); percepção do problema de saúde e procura por cuidados de saúde.

Os dados foram compilados no programa

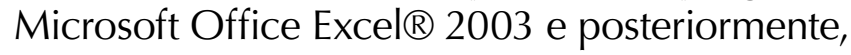
transportados para o programa SPSS, versão 19 e analisados de maneira descritiva em números absolutos epercentuais. O estudo foi aprovado sob parecer n. 007/2011 e CAAE n. 0001.0.268.00011 , obedecendo aos princípios éticos ${ }^{(17)}$.

\section{RESULTADOS}

A idade média dos homens foi de 74,8 anos, com faixa etária entre 72 a 79 (38,9\%); em sua maioria, eram procedentes de Londrina $(83,3 \%)$; declararam ser brancos (53,7\%); católicos (74,4\%) e que coabitavam com a família (89,9\%). A escolaridade, para pouco mais da metade dos homens, foi de até sete anos de estudo (57,4\%). A maioria não trabalhava $(81,5 \%)$ e a fonte de renda era proveniente da aposentadoria pelo Instituto Nacional da Previdência Social (75,9\%).

Quanto à condição do trabalho, 18,5\% trabalhavam sendo $2 \%$ das ocupações na área da construção civil, 2\% na agricultura, como proprietário do próprio negócio $6 \%$, motorista $6 \%$, representante comercial $2 \%$, corretor de imóveis $2 \%$, reciclador de lixo $2 \%$. Ressalta-se que todos os participantes desta pesquisa que não eram autônomos tinham dispensa para realizar o tratamento.

Esses homens eram cuidados em sua maioria (89\%) pelos familiares. Os cuidados identificados foram relacionados com: aspecto financeiro $(17 \%)$, processos domésticos $(28 \%)$, apoio emocional, afetivo e espiritual (22\%) e atividades instrumentais da vida diária (33\%).

Com relação ao comportamento dos homens da presente pesquisa, verificou-se que os alimentos preferidos foram arroz, feijão e massas $(68,5 \%)$, a caminhada foi à atividade física mais praticada $(62 \%)$, receber e visitar parentes e amigos (30\%), ir à igreja, assistir a programas de televisão e ouvir rádio (27\%) foram atividades de lazer mais apreciadas. Quanto ao tempo de uso do tabaco, 28\% afirmaram fumar por mais de 51 anos, com média de 38,4 anos. O uso de bebida alcoólica foi declarado por $67 \%$.

A história familiar demonstrou que $24 \%$ da população do estudo tinham parentes de primeiro grau com diagnóstico de $\mathrm{CaP}$ e $41 \%$ com outros cânceres (mama, útero, pulmão, laringe, esôfago, estomago, vesícula biliar, fígado, intestino, melanoma, leucemia, linfoma, ossos e cérebro). Os sintomas obstrutivos (43\%) e os irritativos (40\%) foram os que mais levaram à procura por cuidado de saúde em 30 dias (55\%). O serviço de saúde de escolha foi para $68,5 \%$ dos pesquisados o de atenção básica, nas Unidades de Saúde (UB) ou em atendimento privado em consultórios. Uma parcela (8\%) demorou mais de seis anos para procurar por assistência e um homem esperou 12 anos, conforme dados da Tabela 1.

Observou-se também nesta Tabela 1 que os exames realizados mais citados, para o rastreamento e controle da doença foram: o teste de Antígeno Prostático Específico (PSA) e o Toque Digital Prostático (TDP) em igual proporção (21\%) e a biópsia guiada por ultrassom transrretal (20\%). Uma grande parcela (38\%) não fazia uso de medicamentos, 43\% utilizavam hormônios e quimioterápicos e 19\% medicamentos específicos para atenuar os efeitos dos sintomas obstrutivos e melhorar o desempenho sexual. A cirurgia mais 
Tabela 1 - História da doença dos portadores de Câncer de Próstata. Londrina-PR-Brasil, 2011

História da doença

\begin{tabular}{|c|c|c|}
\hline História da doença & $\mathbf{n}$ & $\%$ \\
\hline \multicolumn{3}{|l|}{ Antecedente Familiar da doença } \\
\hline Pai & 04 & 7 \\
\hline Irmãos & 9 & 17 \\
\hline Avô/tio/filho & 3 & 5 \\
\hline Outro tipo de câncer & 22 & 41 \\
\hline Desconhece & 8 & 15 \\
\hline Nenhum & 8 & 15 \\
\hline \multicolumn{3}{|l|}{ Primeiros Sintomas } \\
\hline Irritativos & 35 & 40 \\
\hline Obstrutivos & 37 & 43 \\
\hline Relacionados a potencia sexual & 7 & 8 \\
\hline Outros* & 8 & 9 \\
\hline \multicolumn{3}{|l|}{ Tempo de procura por cuidados após $1^{\circ} \mathrm{s}$ sintomas } \\
\hline $1^{\circ}$ mês & 30 & 27 \\
\hline $2^{\circ}$ a $12^{\circ}$ mês & 10 & 27 \\
\hline 2 a 5 anos & 7 & 30 \\
\hline$\geq 6$ anos & 4 & 6 \\
\hline Não Lembra & 3 & 6 \\
\hline \multicolumn{3}{|l|}{ Tipo de serviço de saúde procurado } \\
\hline Atenção Básica & 31 & 16 \\
\hline Atenção secundária & 10 & 12 \\
\hline Atenção terciária & 7 & 16 \\
\hline Consultório particular & 6 & 12 \\
\hline \multicolumn{3}{|l|}{ Exame para rastreamento e controle } \\
\hline PSA (Antigen Specific Prostatic) & 54 & 21 \\
\hline TDP (Toque Digital Prostático) & 54 & 21 \\
\hline Biópsia de próstata guiada por ultrassom transrretal & 51 & 20 \\
\hline Cintilografia Óssea & 30 & 12,5 \\
\hline Tomografia & 34 & 13 \\
\hline Outros & 30 & 12,5 \\
\hline \multicolumn{3}{|l|}{ Terapêutica medicamentosa } \\
\hline Zoladex & 13 & 23 \\
\hline Androcur & 7 & 12 \\
\hline Dietilbestilesterol, ciclofosfamida e decadron & 3 & 5 \\
\hline Quimioterapia endovenosa & 2 & 3 \\
\hline Nenhum & 22 & 38 \\
\hline Outro (doxasozima, finasteride, duomo) & 11 & 19 \\
\hline \multicolumn{3}{|l|}{ Terapêutica cirúrgica e outras } \\
\hline Prostatectomia & 35 & 43 \\
\hline Orquiectomia & 18 & 23 \\
\hline Radioterapia & 23 & 29 \\
\hline Nenhum & 4 & 5 \\
\hline
\end{tabular}

Antecedente Familiar da doença

*Outros sintomas: hematúria, incontinência fecal, dor na região pélvica e hipogástrica. 
realizada (43\%) foi a prostatectomia e $29 \%$ desses homens foram submetidos à radioterapia.

Em relação à história da doença, 37\% dos pesquisados descobriram o $\mathrm{CaP}$ ao realizar exames preventivos, independentemente da presença de sintomas irritativos ou obstrutivos do trato urinário inferior - LUTS (Lower Urinary Tracto Symptoms $)^{(13)}$. O tempo médio de aparecimento dos primeiros LUTS foi de 6,6 anos, o tempo médio de busca por cuidados de saúde foi de 1,4 anos e o tempo médio de diagnóstico do CaP foi de 4,2 anos.

As mudanças que ocorreram na vida desses homens, após o desenvolvimento do $\mathrm{CaP}$, foram: físicas (48\%), emocionais e afetivas (48\%), espirituais (42\%), financeiras (33\%), relacionamento familiar (29\%) e social (24\%).

\section{DISCUSSÃO}

Entre os fatores sociodemográficos de risco, o único considerado bem estabelecido para o desenvolvimento do CaP é a idade. Aproximadamente (62\%) dos casos diagnosticados no mundo ocorreram em homens com 65 anos ou mais ${ }^{(7,18)}$. A população deste estudo se enquadrou nesta faixa etária de risco. O predomínio de homens portadores desta doença que se declararam brancos é semelhante a estudos brasileiros que identificaram também o predomínio de homens brancos ${ }^{(11,19)}$. Contudo, estes dados divergem das estatísticas mundiais, que apontam predominância em indivíduos da raça negra ${ }^{(1,20)}$.

A baixa escolaridade identificada na população pesquisada não chama atenção, pois se trata de indivíduos predominantemente idosos, cujas famílias nas primeiras décadas do século passado, priorizavam a sobrevivência em detrimento da escolarização ${ }^{(12)}$. A baixa escolaridade, associada ao baixo nível socioeconômico, pode ter retardado a busca por cuidados de saúde, principalmente quando o conhecimento sobre o $\mathrm{CaP}$, suas causas, seus sintomas e fatores de risco para a doença é insuficiente ${ }^{(12)}$.

O fato de a maioria residir no município onde realizava o tratamento pode ser considerado fator positivo para a população deste estudo. A facilidade de acesso propicia satisfação e bom vínculo com o serviço de saúde ${ }^{(21)}$. A presença do vínculo entre usuário-profissional-instituição de saúde diminui as barreiras para a continuidade do acompanhamento clínico e propicia o monitoramento dos pacientes mais resistentes e com tendência ao abandono do tratamento ${ }^{(22)}$.

A condição de coabitar com a família pode ser considera como fator de proteção para os homens do presente estudo. Um estudo sobre a masculinidade e práticas de saúde apontou resultado similar ao constatar que $62 \%$ dos homens investigados eram casados, dos quais $53 \%$ relataram que a família contribuiu para o autocuidado, nas situações de agravamento da doença e na estabilidade clínica ${ }^{(23)}$.

A proveniência da renda dos entrevistados em sua maioria foi à aposentadoria pelo INSS. Essa renda, devido à política estabelecida no país, diminui a cada ano, porque a atualização desses valores não corresponde à inflação real ${ }^{(24)}$. Outros trabalhos realizados com pessoas que vivenciam doença crônica retratam essa mesma dificuldade tanto na vida pessoal como na familiar, devido à necessidade de conciliação entre as despesas domésticas e as de transporte e compra de medicamentos ${ }^{(25-26)}$.

Quanto às ocupações, algumas expuseram, por muito tempo, ou ainda expõem esses homens a agentes cancerígenos classificados pela Internacional Agency for Researchon Cancer (IARC) como suspeitos para o desenvolvimento do CaP. Entre estes agentes estão: as poeiras de amianto/abesto e do cádmio; e as aminas aromáticas, que são usadas em fertilizantes, agrotóxicos, tintas de paredes e tinturas para cabelos $^{(27)}$. A associação do tempo prolongado de exposição a agentes cancerígenos, em algumas profissões e o consumo de tabaco, que neste estudo, teve média de 38 anos, pode ter potencializado o risco para o $\mathrm{CaP}$ de $(46 \%)$ dos sujeitos que fumaram ou ainda fumam, uma vez que o consumo de tabaco pode facilitar o desenvolvimento para este tipo de câncer devido à presença de aminas aromáticas presentes no fumo ${ }^{(27)}$.

Entre as mudanças físicas, as relacionadas à sexualidade como alteração na ereção, na qualidade e na frequência ou impotência completa, foram as mais referidas por estes homens. Dados análogos foram identificados em estudos ${ }^{(25-26)}$ no qual revelou que essas e outras modificações decorrentes de câncer 
demandaram enfrentamentos emocionais significativos, provocando carga enorme de estresse para os homens estudados.

O tipo de alimento preferido pelos entrevistados foi o calórico, o que pode contribuir para o excesso de peso. A obesidade advinda desses alimentos pode influenciar, potencialmente, o desenvolvimento do $\mathrm{CaP}$, por provocar algumas alterações como resistência insulínica, alteração no perfil lipídico, inflamação, menor concentração de PSA, diminuição dos andrógenos e aumento de estrógenos ${ }^{(28)}$.

Em estudo realizado na Austrália, havia uma previsão de redução de 4.882 casos de CaP até 2025, se os homens praticarem exercícios físicos, dieta adequada e reduzirem a obesidade ${ }^{(28)}$. A realização de atividades de lazer e exercícios físicos foi frequente na população de estudo e pode estar relacionada ao tempo disponível em função da aposentadoria. Este fato evidencia que houve busca de melhoria na qualidade de vida e na condição de saúde por parte dos homens portadores de CaP. Por outro lado, uma parcela expressiva dos homens relatou alguns comportamentos de risco à saúde, como o consumo de bebida alcoólica e o uso de tabaco.

A história familiar aponta que (24\%) dos homens investigados apresentavam 2,2 vezes mais risco para desenvolver o $\mathrm{CaP}$ por terem parentes de primeiro grau com a doença ${ }^{(7)}$. O conhecimento prévio da relação da doença com antecedentes familiares, dos sintomas do trato urinário inferior e dos fatores predisponentes, poderia ter favorecido a prática do autocuidado entre esses homens, pois o tempo médio da procura por cuidados de saúde, para (45\%) foi superior a 30 dias, atingindo até 12 anos. Este quadro evidencia que os mesmos não valorizaram os sintomas principalmente os obstrutivos, que predominaram sobre os demais. A incidência do CaP em pacientes que desenvolvem retenção urinária aguda tem sido estimada entre 13 a 25\% ${ }^{(7)}$.

Os exames realizados foram os indicados para o rastreamento do CaP: o toque retal, a dosagem do PSA total e a ultrassonografia pélvica ou prostática transrretal utilizada para a realização de biópsia de próstata ${ }^{(8,29)}$. A introdução da dosagem do PSA contribuiu para a redução da resistência masculina ao diagnóstico do $\mathrm{CaP}$, o que não se verifica com o toque retal, que apesar de ser uma medida preventiva de baixo custo, é um procedimento que mexe com o imaginário masculino a ponto de afastar inúmeros homens da prevenção do câncer de próstata ${ }^{(12)}$.

Os serviços de Atenção Básica foram referenciados como o primeiro atendimento. Esse resultado distingue um diferencial no comportamento destes indivíduos. Estudos revelam que homens procuram mais os serviços de saúde especializados quando do motivo de doença ${ }^{(12,22)}$.

As terapêuticas utilizadas corresponderam às indicadas nas diretrizes de tratamento para o $\mathrm{CaP}$ e envolveram: uso de medicamentos, cirurgias e radioterapia, ou somente o acompanhamento clínico, associado ou não a outros procedimentos, dependendo do estágio da doença ${ }^{(7,29)}$.

Frente ao exposto, os dados abalizam que os profissionais de saúde participam da implementação das ações constituídas pelas políticas de saúde voltadas à assistência ao homem, quando realizam o rastreamento da doença e adotam medidas de prevenção e promoção da saúde. Neste contexto, é fundamental à enfermagem conhecimento dos tratamentos oncológicos vigentes para o desenvolvimento de atividades educativas que contribuam para a adesão do paciente ao tratamento ${ }^{(30)}$.

\section{CONSIDERAÇÕES FINAIS}

Este estudo aponta que os homens, demoraram mais de um ano para buscar por cuidados de saúde após o aparecimento dos primeiros sintomas do trato urinário inferior, reforçando que os serviços de saúde precisam desenvolver estratégias de informação para o binômio homemfamília sobre a importância de uma atitude positiva em relação à prevenção de doenças.

Muitos profissionais de saúde adotaram em sua práxis ações voltadas para a detecção precoce do $\mathrm{CaP}$, pois grande parcela da população pesquisada foi diagnosticada por meio de exames preventivos realizados na US.

Ressalta-se a necessidade dos órgãos públicos ou políticas públicas locais, estaduais e nacionais aprimorarem os programas de saúde no sentido de melhorar a qualidade de vida da população masculina, pois o aumento da expectativa de vida constitui uma realidade brasileira.

Embora os objetivos do estudo tenham sido 
atingidos há limites devido ao método adotado, isto é, por se tratar de um estudo transversal que foi aplicado em um determinado tempo e desenvolvido apenas com uma parcela dos pacientes com CaP de um Hospital Escola, não podendo ser generalizado.

Entretanto, acredita-se que o estudo contribuiu para o avanço do conhecimento, visto que, ainda é incipiente no Brasil estudos específicos do gênero masculino. Assim, sugere-se que outros estudos sejam realizados nesta mesma instituição, bem como outras, com a finalidade de promover a saúde dos homens, prevenir as doenças e diminuir os agravos à saúde.

\section{REFERÊNCIAS}

1. IARC. International Agency for Research on Cancer, World Health Organization [Internet]. 2012 [acesso em 12 abr 2013]. Study finds prostate cancer increasing in most countries. Rates remain highest in highest income regions of the world. Press Release, n. 209. Disponível: http:// www.iarc.fr/en/media-centre/pr/2012/pdfs/pr209_E.pdf

2. IARC. International Agency for Research on Cancer. World Health Organization [Internet] 2012 [acesso em 16 jan 2014]. GLOBOCAN 2012. Estimated cancer Incidence, Mortality and Prevalence Worldwide in 2012. Disponível: http://globocan.iarc.fr/Default.aspx

3. IARC. International Agency for Research on Cancer. World Health Organization [Internet] 2008 [acesso em 01 mar 2013]. GLOBOCAN 2008. Prostate cancer incidence, mortality and prevalence world wide in 2008. Disponível: http://www.iarc.fr/en/media-centre/ iarcnews/2010/globocan2008.php

4. INCA. Instituto Nacional do Câncer. Coordenação Geral de Ações e Estratégias.

5. INCA. Instituto Nacional do Câncer. Estimativa 2014: incidência de câncer no Brasil. Rio de Janeiro: Inca; 2014. [Internet] [acesso em 14 jan 2014]. Disponível: http://www.inca.gov.br/estimativa/2014/

6. Crawford ED. Understanding the epidemiology, natural history, and key pathways involved in prostate cancer. Urology. 2009;73(5 Suppl):4-10. [acesso em 01 set 2011] Disponível: http://www.sciencedirect.com/ science/article/pii/S0090429509002842

7. Correa FM. Epidemiologia. In: Guimarães RQ. Manual de oncologia. $3^{\text {a }}$ ed. São Paulo: BBS; 2008. p.73-88.

8. Rhoden EL, Averbeck MA. Câncer de próstata localizado. Rev. Assoc. Med. Rio Gd. Sul. [Internet] 2010;54(1) [acesso em 15 ago 2013]. Disponível: http:// www.amrigs.org.br/revista/54-01/20-488_cancer_de_ prostata.pdf

9. Dall'Oglio MF, coordenador. Diretrizes de câncer de próstata. Rio de Janeiro: SBU; 2011. 92p. [Internet] [acesso 15 jan 2014]. Disponível: http://sbues.org.br/ diretrizes/cancer_prostata.pdf

9. ACS. American Cancer Society. Prostate Cancer: Early Detection. [Internet] 2014 [acesso 13 jan 2014]. Disponível: http://www.cancer.org/ cancer/prostatecancer/moreinformation/ prostatecancerearlydetection/prostate-cancer-earlydetection-toc

10. Gonçalves IR, Padovani C, Popim RC. Caracterização epidemiológica e demográfica de homens com câncer de próstata. Ciênc. saúde colet. 2008;13(4):1337-42.

11. Vieira LJES, Santos ZMSA, Landim FLP, Caetano JN, Neta CAS. Prevenção do câncer de próstata na ótica do usuário portador de hipertensão e diabetes. Ciênc. saúde colet. 2008;13(1):145-52.

12. Gomes R, Nascimento EF, Rebello LEFS, Araújo FC. As arranhaduras da masculinidade: uma discussão sobre o toque retal como medida de prevenção do câncer prostático. Ciênc. saúde colet. 2008;13(6):1975-84.

13. Paschoalick RC, Lacerda MR, Centa ML. Gênero masculino e saúde. Cogitare enferm. [Internet] 2006;11(1) [acesso 7 dez 2013]. Disponível: http:// ojs.c3sl.ufpr.br/ojs2/index.php/cogitare/article/ view/5979/4279

14. Fontes WD, Barbosa TM, Leite MC, Fonseca RLS, Santos LCF, Nery TCL. Atenção à saúde do homem: interlocução entre ensino e serviço. Acta Paul. Enferm. 2011; 24(3):430-3.

15. Ministério da Saúde (BR). Secretaria de Atenção à Saúde [Internet]. Brasília; 2008. [acesso em 21 dez 2012]. Política nacional de atenção integral à saúde do homem: princípios e diretrizes. Disponível: http:// dtr2001.saude.gov.br/sas/PORTARIAS/Port2008/PT09-CONS.pdf.

16. Brasil. Lei no 10.289, de 20 de setembro de 2001. Institui o Programa Nacional de Controle do Câncer de Próstata. [Internet] [acesso em 11 jan 2014]. Disponível: http://www.planalto.gov.br/ccivil_03/leis/ leis_2001/l10289.htm

17. Ministério da Saúde (BR). Conselho Nacional e Saúde. Comissão Nacional de Ética em Pesquisa. Resolução $n^{0} 169 / 96$. Dispõe sobre as diretrizes e normas regulamentadoras de pesquisas envolvendo seres humanos. Brasília: MS; 1996.

18. INCA. Instituto Nacional do Câncer. Coordenação Geral Ações Estratégicas. Coordenação de Prevenção e Vigilância. Estimativa 2012: incidência de câncer no 
Brasil. Rio de Janeiro: INCA; 2011.118p

19. Paiva EP, Motta MCS, Griep RH. Conhecimentos, atitudes e práticas acerca da detecção do câncer de próstata. Acta Paul. Enferm. 2010;23(1):88-93

20. Romero FR, Romero AW, Almeida RM, Tambara Filho $R$. The prevalence of prostate cancer in Brazil is higher in Black men than in White men: systematic review and meta-analysis. Int Braz J Urol 2012;38(4):440-7. [acesso 15 jan 2014]. Disponível: http://www.brazjurol. com.br/july_august_2012/Romero_440_447.pdf

21. Dall'agnol CM, Lima MADS, Ramos DD. Fatores que interferem no acesso de usuários a um ambulatório básico de saúde. Rev. Eletr. Enf. [Internet] 2009;11(3) [acesso em $18 \mathrm{dez}$ 2012]. Disponível: http://www.fen. ufg.br/revista/v11/n3/v11n3a27.htm

22. Palermo PU, Kuehn BM. Veterans health system cited by experts as a model for patient-centered care. JAMA. 2012;307(5):442-3. . http://jama.jamanetwork.com/ article. aspx?articleid $=1228886$

23. Nascimento ARA, Trindade ZA, Gianordoli-Nascimento IF, Pereira FB, Silva SATC, et al. Masculinidades e práticas de saúde na região metropolitana de Belo Horizonte - MG. Saúde soc. [Internet] 2011;20(1) [acesso em 10 jan 2013]. Disponível: http://dx.doi. org/10.1590/S0104-12902011000100020

24. Bulla LC, Kaefer CO. Trabalho e aposentadoria: as repercussões sociais na vida do idoso aposentado. Rev. Virtual Textos Contextos. [Internet] 2003;2(2) [acesso em 15 maio 2013]. Disponível: http:// revistaseletronicas.pucrs.br/ojs/index.php/fass/article/ viewFile/957/737

25. Cardoso CCL, Rosalini MHP, Pereira MTAM A. O cuidar na concepção dos cuidadores: um estudo com familiares de doentes crônicos em duas unidades de saúde da família de São Carlos-SP. Serv. soc. rev. 2010;13(1):24-42. http://www.uel.br/revistas/uel/index. php/ssrevista/article/view/8732/9090

26. Miliorini JP, Fernandes MV, Decesaro MN, Marcon SS. A família no contexto hospitalar: apreendendo os anseios e expectativas relacionadas com doença crônica. Rev. Rene. 2008;9(3):81-91.

27. INCA. Instituto Nacional do Câncer. Coordenação de Prevenção e Vigilância.Vigilância do câncer relacionado ao trabalho e ao ambiente. $2^{\text {a }}$ ed. Rio de Janeiro: INCA; 2010.

28. Baade PD, Meng X, Sinclair C, Youl P. Estimating the future burden of cancers preventable by better diet and physical activity in Australia. MJA. 2012;196(5):337-28-9.

29. Campos HLM, Dias FMV, Moraes SC, Vargas SC. Aspectos culturais que envolvem o paciente com diagnóstico de neoplasia de próstata: um estudo na comunidade. Rev. bras. cancerol. 2011;57(4):493-501. [acesso em mai 2012] Disponível: http://www.inca.gov.br/rbc/n_57/ v04/pdf/05_artigo_aspecto_culturais_envolvem_ paciente_diagnostico_neoplasia_prostata.pdf

30. Lira ALBC, Sá ID, Nogueira ILA, Medeiros MDC, Fernandes MICD, et al. Integridade da pele em idosos: revisão de literatura segundo as cartas de promoção da saúde. Cogitare enferm. 2012;17(4):767-74. (http://ojs.c3sl.ufpr.br/ojs2/index.php/cogitare/article/ viewFile/30389/19664) 